\title{
Complications with rotavirus: A single center experiences
}

\author{
Bilge Aldemir-Kocabaş ${ }^{1}$, Adem Karbuz ${ }^{1}$, Halil Özdemir ${ }^{1}$, Tuğçe Tural-Kara ${ }^{1}$, Anıl Tapısız ${ }^{1}$ \\ Nurşen Belet ${ }^{1}$, Haluk Güriz ${ }^{2}$, Ergin Çiftçi ${ }^{1}$, Erdal İnce ${ }^{1}$ \\ ${ }^{1}$ Division of Pediatric Infectious Diseases, Department of Pediatrics, ${ }^{2}$ Department of Clinical Microbiology, Ankara \\ University Faculty of Medicine, Ankara, Turkey. \\ E-mail:drbaldemir@yahoo.com/drbaldemir@gmail.com \\ Received: 13th January 2017, Accepted: 3rd February 2017
}

SUMMARY: Aldemir-Kocabaş B, Karbuz A, Özdemir H, Tural-Kara T, Tapısız $\mathrm{A}$, Belet $\mathrm{N}$, Güriz $\mathrm{H}$, Çiftçi $\mathrm{E}$, İnce $\mathrm{E}$. Complications with rotavirus: A single center experiences. Turk J Pediatr 2016; 58: 602-608.

Rotavirus is an important morbidity and mortality agent among previously healthy infants. Rotavirus gastroenteritis (RG) causes severe fluid and electrolyte imbalance and development of fatal complications during course of the disease, if untreated. Although there are few case reports in the literature regarding septicemia after RG, it is one of the most important complications of the disease. The main reason of septicemia is unknown but it can be associated with severe consequences such as septic shock, endocarditis, and even death. If complications are taken into consideration on the course of disease, morbidity and mortality can be reduced. Herein, we evaluated the clinical characteristics, incidence and types of the complications during RG in this retrospective study. We evaluated a total of 276 patients (164 male, 112 female) diagnosed with RG between January 2006 and February 2013 at the department of Pediatric Infectious Disease. Male to female ratio was 1.5 and the median age at admission was 12 months (1-102 months). None of the patients had a history of Rotavirus immunization. While overall complication rate was $44.2 \%$, the most common complication related RG was electrolyte imbalance and the second common complication was septicemia $(32.6 \%$ and $6.5 \%$, respectively). Klebsiella, E. coli and Candida species were the most common isolated microorganisms from the blood cultures.

Rotavirus gastroenteritis is still an important cause of morbidity in children which can cause serious complications such as sepsis. Lack of rotavirus vaccine in our national immunization program might contribute to the related complications and prolonged hospitalization, health care expenses and family work loss, as well.

Key words: complications, rotavirus, septicemia, vaccine.

Rotavirus gastroenteritis (RG) is the most common cause of diarrhea among infants and young children in all regions of the world ${ }^{1-3}$. RG is responsible for severe fluid and electrolyte loss and may cause life-threatening complications in children who have no prompt access to rehydration ${ }^{1}$. Despite low mortality rates in developed countries, rotavirus is a major cause of morbidity and health care costs for these countries. Easy access to health care services, increased use of oral rehydration solutions and better nutrition may be responsible factors from lower mortality rates in these regions. However, the incidence and severity of rotavirus infections has declined significantly in countries that have added rotavirus vaccine to routine childhood immunization policies 4,5 . The considerable causes of death from rotavirus gastroenteritis are dehydration and severe electrolyte abnormalities leading to cardiovascular failure. Some complications can occur during the course of illness, if untreated. These complications are; hypotonic or hypertonic dehydration, respiratory infections, necrotizing enterocolitis (especially in preterm infants), pneumatosis intestinalis, hepatic abscess, hepatitis, hemophagocytic lymphohystiocytosis, Reye like syndrome, acute myositis, sepsis, myocarditis, seizures 
and meningoencephalitis. Also, a relationship between rotavirus and autoimmune diseases such as celiac disease has been reported ${ }^{1-3,6-9}$. Secondary capillary leak syndrome in an infant, have been described related with $\mathrm{RG}^{10}$. The underlying causes of some complications due to RG which are more seen in some children remain unknown.

In this study we aimed to assess the clinical characteristics and complications in patients diagnosed with RG in our clinic.

\section{Material and Methods}

We evaluated medical records of patients diagnosed with RG and hospitalized in our clinic between January 2006 and February 2013, retrospectively. The following data were collected for all patients: age at admission, gender, month and year of admission, rotavirus vaccination history, length of hospitalization, duration of fever, vomiting and diarrhea, accompanying diseases, serum sodium, potassium and creatinine levels, blood urea nitrogen and serum creatinine levels, C-reactive protein and serum leukocyte counts, body temperature at admission, number of vomiting and diarrhea per day, degree of dehydration, complications, results of blood and urine cultures, source of infection (nosocomial or community acquired). Collection of stools was performed within 3 days after admission and nosocomial RG is defined as infections occurred after 72 hours of admission. Serum sodium and potassium levels were considered hyponatremia $(<135 \mathrm{mmol} / \mathrm{L})$ or hypernatremia $(\geq 145$ $\mathrm{mmol} / \mathrm{L})$, hypokalemia $(<3.5 \mathrm{mEq} / \mathrm{L})$ or hyperkalemia $(>5.5 \mathrm{mEq} / \mathrm{L})^{3}$. Rotavirus antigen (RVA) in stool samples was detected by ELISA and only cases with positive for RVA in stool were included to the study.

Statistical analyze was performed using SPSS 15.0 software. Quantitative data were expressed as mean \pm SD or median (range) where appropriate. We used "Student t test" for parametric assumptions and "Mann-Whitney-U test" for non- parametric assumptions. For comparison of categorical data "Chi-square test" and correlations of the variables "Pearson Spearman" was used.

\section{Results}

A total of 276 patients (164 male, 112 female) diagnosed with RG were enrolled in this study. Male to female ratio was 1.5 and the median age at admission was 12 months (1102 months). Nineteen of the cases (6.9\%) had nosocomial RG. RG was most commonly detected in November and December (Fig. 1). There were no significant differences between distributions of the cases throughout the years. None of the patients had received rotavirus vaccine before. The average durations of fever, vomiting and diarrhea were $2.3 \pm 1.9 ; 2.6 \pm$ 1.7 and $7.0 \pm 2.4$ days, respectively. The mean disease day at admission was $3.1 \pm 1.7$ days. $54 \%$ of cases had mild dehydration (loss of $\% 5$ of total body weight) and $46 \%$ of patients had moderate-to-severe dehydration (loss of $>\% 5$ of total body weight). Demographic, clinical and laboratory data of the patients was shown in Table I. Hyponatremia in 66 cases $(23.9 \%)$, hypernatremia in 9 cases $(3.3 \%)$, hypokalemia in 23 patients $(8.3 \%)$ and hyperkalemia in 6 cases $(2.2 \%)$ were present at admission.

The total complication rate was $44.2 \%$ and septicemia was the second common complication (6.5\%) following fluid and electrolyte imbalance (32.6\%). Klebsiella, E. coli and Candida species were the most common isolated microorganisms from the blood cultures. In some patients, unusual microorganisms were isolated from the blood cultures as shown in Table II. A 22-month-old patient was admitted to our clinic with complaints of fever and diarrhea. He had an atypical disease course and finally was diagnosed with $S$. aureus bacteremia, pyomyositis and endocarditis as complications of RG. At the end of an appropriate antibiotic regimen, he was successfully treated and discharged. Another patient was a 16-month-old girl with mitochondrial disease who was admitted to our clinic with RG. Her fever did not resolve on the fifth day of the disease and her blood culture was positive for Leuconostoc mesenteroides on the sixth day of the disease. She was admitted to pediatric intensive care unit due to septic shock and septic shock resolved with prompt and appropriate treatment. Subsequent blood cultures remained negative during follow-up. In another patient with a neurologic disorder, Candida lusitaniae was obtained from the blood culture on the fifth day of the RG. He was treated with antifungal treatment successfully without any sequelae.

Five patients with septicemia needed pediatric 


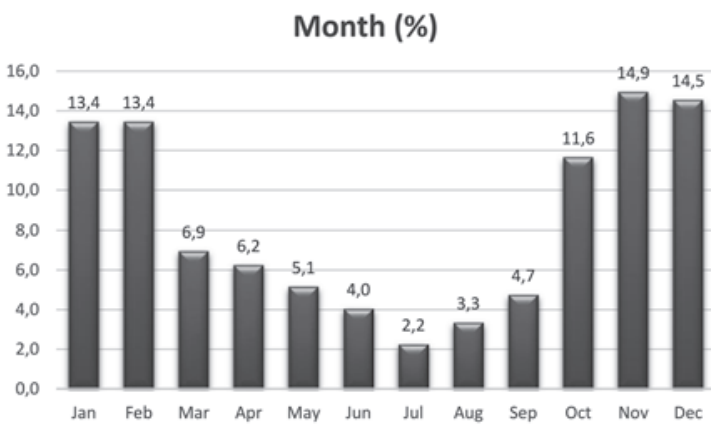

Fig. 1. Distribution of the cases with rotavirus gastroenteritis by months.

intensive care unit admission due to septic shock and disseminated intravascular coagulation. None of them had any sequelae or mortality with appropriate and prompt treatment on the follow up. Other complications include seizures $(2.2 \%)$, intussusceptions $(3.3 \%)$, aseptic meningitis $(0.7 \%)$, capillary leak syndrome, toxic hepatitis, thrombocytopenia, neutropenia, secondary lactose intolerance (Table III). While there were respiratory symptoms in 51 patients (18.5\%) prior to the admission, bronchiolitis which required treatment during course of the disease developed in 14 cases $(5.1 \%)$. The duration of hospitalization was significantly higher in complicated cases than those of uncomplicated cases $(\mathrm{p}<0.001)$.

Accompanying diseases were present in $15.6 \%$ of the cases. These were cerebral palsy, Alagille syndrome, congenital heart defects, Down syndrome, neuromotor retardation, epileptic disorders, mitochondrial disease, nephrotic syndrome, Trisomy 9, nephrolithiasis, and malnutrition. Laboratory tests were as following: median leukocyte count was 9,300/

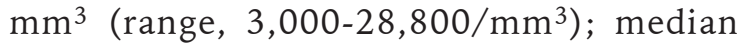
C-reactive protein level was $0.3 \mathrm{mg} / \mathrm{L}$ (range, 0.1-144 mg/L); median blood urine nitrogen level was $17 \mathrm{mg} / \mathrm{dL}$ (range, 1-106 mg/dL); and mean serum creatinine level was $0.3 \pm 0.1 \mathrm{mg} /$ $\mathrm{dL}$. There were no difference between boys and girls in terms of complication rate $(\mathrm{p}=0.54)$. Also, there was no difference in the duration of diarrhea and vomiting between complicated and uncomplicated patients $(\mathrm{p}=0.11$ and $\mathrm{p}=0.94$, respectively). The duration of fever in patients with complications was significantly longer than those with uncomplicated cases $(p=0.04)$. Body temperatures of the complicated patients on

Table I. Demographic, Clinical and Laboratory Data of the Patients with Rotavirus Gastroenteritis

Median age, months (range)

Gender $(\mathrm{M} / \mathrm{F}), \mathrm{n} / \mathrm{n}$

Nosocomial rotavirus gastroenteritis $\mathrm{n}(\%)$

Duration of fever (day \pm ), mean \pm SD

Duration of vomiting (day), mean \pm SD

Duration of diarrhea (day), mean \pm SD

Mean disease day at admission, mean \pm SD

Mild dehydration, n (\%)

Moderate/severe dehydration, n (\%)

Respiratory symptoms, n (\%)

Duration of hospitalization (day), mean \pm SD

Median CRP (mg/L), (range)

Median leucocyte $\left(/ \mathrm{mm}^{3}\right)$ (range)

Median blood urea nitrogen (mg/dl), (range)

Creatinine $(\mathrm{mg} / \mathrm{dl})$, mean $\pm \mathrm{SD}$

Median sodium level (mEq/L), (range)

Median potassium level (mEq/L), ( range)
$12(1-102)$

$164 / 112$

$19(6.9)$

$$
\begin{aligned}
& 2.3 \pm 1.9 \\
& 2.6 \pm 1.7 \\
& 7.0 \pm 2.4 \\
& 3.1 \pm 1.7 \\
& 148(54) \\
& 128(46) \\
& 51(18.5) \\
& 5.18 \pm 2.5
\end{aligned}
$$

$0.3(0.1-144)$

$9,300(3,000-28,800)$

17 (1-106)

$0.3 \pm 0.1$

137 (123-170)

$4.2(2.3-7.2)$ 
admission were also higher $(\mathrm{p}=0.03)$. There was no significant correlation between the day of admission and complication risk $(\mathrm{p}=0.36)$. There was no significant difference between nosocomial RG and non-nosocomial RG patients in terms of complication risk, bacteremia and electrolyte imbalance $(\mathrm{p}=0.50, \mathrm{p}=1.000$, and $\mathrm{p}=0.54$, respectively). Complications between two groups who had co-morbid disease or not, were similar $(p=0.31)$. Also, the durations of fever, diarrhea and vomiting were not significantly different between these groups $(p>0.05)$. Distribution of complications between the months of the year did not show a significant difference $(p=0.08)$.

\section{Discussion}

Rotavirus is responsible for the majority of hospitalizations due to gastroenteritis in children especially under five years of age. It causes an annual peak in pediatric hospitalizations especially during the cooler months of the year. In our study, we found that RG was most commonly detected in November and December. The reason of seasonality of RG remains unknown ${ }^{1-3}$.

The most common complication of the illness is electrolyte imbalance which is thought to be due to the viral enterotoxin responsible for malabsorption related to mucosal damage and depression of disaccharidases. The severity of diarrhea correlates with the degree of mucosal damage ${ }^{1-3}$. In a study examining the complications of RG in hospitalized children, 1.7 percent had hypertonic dehydration requiring intensive care and 9.1 percent had hypertonic dehydration ${ }^{11}$. Hyponatremic and hypokalemic dehydrations were the most common complications in our cases.

The second most common complication was septicemia in which the exact mechanism is unknown and it is believed that infected enterocytes become more vulnerable to bacterial

Table II. Data of the Patients with Septicemia

\begin{tabular}{cccccccc}
\hline Patient & $\begin{array}{c}\text { Age (Month) } \\
\text { /Sex }\end{array}$ & $\begin{array}{c}\text { Comorbid } \\
\text { disease }\end{array}$ & $\begin{array}{c}\text { Duration } \\
\text { of fever } \\
\text { (days) }\end{array}$ & $\begin{array}{c}\text { Duration } \\
\text { of diarrhea } \\
\text { (days) }\end{array}$ & $\begin{array}{c}\text { Duration } \\
\text { of vomiting } \\
\text { (days) }\end{array}$ & Microorganism & Treatment \\
\hline 1 & $12 / \mathrm{M}$ & - & 4 & 7 & 5 & K.pneumoniae & Ceftriaxone \\
2 & $60 / \mathrm{F}$ & - & 4 & 5 & 2 & K.pneumoniae & Ceftriaxone \\
3 & $2 / \mathrm{M}$ & - & 8 & 8 & 3 & K.pneumoniae & Meropenem \\
4 & $3 / \mathrm{F}$ & $+(\mathrm{CHD})$ & 4 & 9 & 1 & K.pneumoniae & Ceftriaxone \\
5 & $8 / \mathrm{F}$ & - & 4 & 6 & 2 & K.pneumoniae & Meropenem \\
6 & $9 / \mathrm{M}$ & - & 5 & 10 & 3 & K.oxytoca* & Meropenem \\
7 & $4 / \mathrm{M}$ & - & 4 & 14 & 2 & E.coli & Ceftriaxone \\
8 & $17 / \mathrm{M}$ & $+(\mathrm{DS})$ & 5 & 4 & 2 & E.coli & Ceftriaxone \\
9 & $2 / \mathrm{F}$ & - & 5 & 9 & 1 & E.coli & Ceftriaxone \\
10 & $16 / \mathrm{M}$ & $+(\mathrm{NS})$ & 4 & 8 & 2 & E.coli & Ceftriaxone \\
11 & $36 / \mathrm{M}$ & $+(\mathrm{ND})$ & 5 & 3 & 2 & C.albicans & Fluconasole \\
12 & $5 / \mathrm{M}$ & - & 4 & 11 & 4 & C.albicans & Amph-B \\
13 & $2 / \mathrm{M}$ & $+(\mathrm{ND})$ & 3 & 6 & 2 & C.lusitaniae & Caspofungin \\
14 & $18 / \mathrm{M}$ & - & 4 & 8 & 2 & P.aeruginosa & Meropenem \\
15 & $14 / \mathrm{F}$ & - & 4 & 7 & 2 & E.cloaca & Ceftriaxone \\
16 & $16 / \mathrm{F}$ & $+(\mathrm{MD})$ & 9 & 9 & 3 & L.mesenteroides & Ceftriaxone,A \\
17 & $22 / \mathrm{M}$ & - & 8 & 5 & 1 & S.aureus & SAM,C,G \\
18 & $34 / \mathrm{F}$ & - & 4 & 7 & 3 & S.pneumoniae & Ceftriaxone \\
\hline
\end{tabular}

* Extended spectrum beta lactamases (ESBL) positive, Admission to Pediatric Intensive Care CHD: congenital heart disease; DS: Down syndrome; NS: nephrotic syndrome; ND: neurologic disease; MD: mitochondrial disease; Amph-B: lyposomal amphoterisin B; A: ampicillin; SAM: ampicillin-sulbactam; C: clindamycie; G: gentamicin 
invasion as a result of intestinal epithelium dysfunction caused by rotavirus ${ }^{2,3,12}$. In our study, although we did not observe significant differences in general clinical characteristics of RG in our patients compared to previous studies in the literature ${ }^{13-15}$, we detected a high rate of septicemia $(6.5 \%)$ as a complication. The most common isolated microorganisms from the blood cultures were Klebsiella, E. coli and Candida species. Early diagnosis of bacteremia and candidemia during course of RG and starting the appropriate treatment is of critical importance.

There are only few case reports in the literature currently regarding septicemia after RG 12, 16-19. So, exact incidence of septicemia during the disease is not known. Carneiro et al. ${ }^{17}$ reported a child who had RG complicated with septic shock due to ESBL positive E. coli. Kashiwagi et al. ${ }^{19}$ also described Klebsiella sepsis in the course of RG in an infant. Moreover, Nakataniet al. ${ }^{16}$ reported a case with RG which was complicated with sepsis due to Klebsiella pneumoniae, too.
Gonza'lez-Carretero et al. ${ }^{20}$ published a study in 2006 which had evaluated 891 children with RG. They reported bacteremia in only two infants (S. viridans ve E. cloaca). Also, they mentioned a total of 8 cases in the literature in whom bacteremia had occurred during course of RG. Our bacteremia incidence was higher than some of recent studies 13,20 . It might be related to the serotypes being responsible for more severe infections in our region. Although it is only a speculative reason due to lack of serotyping in our study, Durmaz et al. recently reported a Rotavirus surveillance study in Turkey $^{21}$. It was conducted in the same time period of our study and they found that G9P8 was the most common serotype. The same $\mathrm{G} / \mathrm{P}$ combination was found to be associated with more severe disease in another study conducted by Linhares et al. ${ }^{22}$.

However, more comprehensive and multicenter studies are required to determine the causative serotypes for the severe disease forms in our country. There is no case with S. aureus, Candida

Table III. Type and Rate of the Complications of the Cases

\begin{tabular}{lc}
\hline Type of complication & $\mathrm{n}(\%)$ \\
\hline Electrolyte imbalance & $90(32.6)$ \\
$\quad$ Hypernatremia & $9(3.3)$ \\
$\quad$ Hyponatremia & $66(23.9)$ \\
$\quad$ Hyperkalemia & $6(2.2)$ \\
$\quad$ Hypokalemia & $23(8.3)$ \\
Septicemia & $18(6.5)$ \\
Bronchiolitis & $14(5.1)$ \\
Intussusception & $9(3.3)$ \\
Convulsion & $6(2.2)$ \\
Aseptic meningitis & $2(0.7)$ \\
IE and PM & $1(0.4)$ \\
CLS & $1(0.4)$ \\
Paralytic ileus & $1(0.4)$ \\
SLI & $1(0.4)$ \\
Septic shock, DIC & $5(1.8)$ \\
Neutropenia & $1(0.4)$ \\
Thrombocytopenia & $1(0.4)$ \\
Vasculitis & $1(0.4)$ \\
Toxic hepatitis & $1(0.4)$ \\
\hline E
\end{tabular}

IE: infective endocarditis; PM: pyomyositis; CLS: capillary leak syndrome; SLI: secondary lactose intolerance; DIC: disseminated intravascular coagulation 
lusitaniae and Leuconostoc mesenteroides septicemia which had been reported previously in the literature. Although gram negative bacteremia has been stated as a complication in RG, $S$. aureus bacteremia and endocarditis have not been reported in the literature before. We had reported this case as a case report. ${ }^{23}$ It was striking data that we had observed a variety of complications of RG, even critically ill cases among 276 patients; none of them had a rotavirus vaccination history, unfortunately. Although our national immunization program does not include rotavirus vaccine, having a low socioeconomic status probably played an important role for lack of voluntary vaccination in our study population.

RG has two phases during the disease course; vomiting and fever are forefront at the first phase which lasts approximate 1-2 days, at the second phase of the disease diarrhea becomes apparent while fever and vomiting cease. During the second phase, diarrhea lasts about a week, rarely 10 days ${ }^{1-3}$. If fever persists or comes out again especially after the third day of the disease, secondary bacteremia should be suspected and all cultures should obtain immediately. Therefore, if an infant has septic appearance and abdominal distension in addition to persistent fever, an antibiotic treatment should be started after taking all cultures. RG not only gives rise to bacteremia and sepsis but also can cause some complications such as severe dehydration, electrolyte imbalance, neurologic disorders (cerebellitis and encephalitis), hepatitis, necrotizing enterocolitis (in preterm infants), respiratory infections and myocarditis ${ }^{1-3}, 6-9,24$. We reported a secondary capillary leak syndrome related RG previously ${ }^{10}$. Sweet syndrome and celiac disease are other reported complications in these patients ${ }^{1,2,9}$. Accompanying respiratory system disorders such as bronchiolitis also can occur during or just before RG. Transmission through airborne droplets has been hypothesized because of rapid seasonal transmission through populations, but it has not been proven ${ }^{1}$. In our current study, we found the respiratory symptoms incidence as $18.5 \%$. Its reason is unclear.

The present study has several limitations. Firstly, the study design was retrospective. Second, we studied a relatively small number of patients, especially in subgroups, which may affect the power of the study. Third, we could not compare the complications with another gastroenteritis agent other than rotavirus. Lastly, we did not perform a serotype study for rotavirus.

In conclusion, RG is still an important cause of morbidity in children which can cause serious complications such as sepsis. With the frequency of the disease, the serious complications leading to longer duration of hospitalization, high health care costs and family work loss are taken into consideration besides morbidity and mortality. The use of efficient RG vaccine may be of value.

\section{REFERENCES}

1. Staat MA, McNeal MM, Bernstein DI. Rotavirus. In: Cherry JD, Harrison GJ, Kaplan SL, et al (eds). Feigin and Cherry's Textbook of Pediatric Infectious Diseases (7th ed). Philadelphia: W.B. Saunders, 2014: 2176-2195.

2. Yen C, Cortese MM. Rotaviruses. In: Long SS, Pickering LK, Prober CG (eds). Principles and Practice of Pediatric Infectious Diseases (4th ed). Philadelphia: Elsevier Saunders, 2012: 1094-1097.

3. Bass DM. Rotaviruses, Caliciviruses, and Astroviruses. In: Kliegman RM, Behrman RE, Jenson HB (eds). Nelson Textbook of Pediatrics (19 $9^{\text {th }}$ ed). Philadelphia, Elsevier Saunders, 2011: 1134-1137.

4. Giaquinto C, Dominiak-Felden G, Van Damme P, et al. Summary of effectiveness and impact of rotavirus vaccination with the oral pentavalent rotavirus vaccine: A systematic review of the experience in industrialized countries. Hum Vaccin 2011; 7: 734-748.

5. Jiang V, Jiang B, Tate J, Parashar UD, Patel MM. Performance of rotavirus vaccines in developed and developing countries. Hum Vaccin 2010; 6: 532-542.

6. Thompson MJ, Gowdie PJ, Kirkwood CD, Doherty RR, Fahey M. Rotavirus cerebellitis: new aspects to an old foe? Pediatr Neurol 2012; 46: 48-50.

7. Iwasa T, Matsubayashi N. Protein-loosingenteropathy associated with rotavirus infection in an infant. World J Gastroenterol. 2008; 14: 1630-1632.

8. Kobayashi S, Negishi Y, Ando N, et al. Two patients with acute rotavirus encephalitis associated with cerebellar signs and symptoms. Eur J Pediatr 2010; 169: 1287-1291.

9. Makis A, Stavrou S, Chaliasos N, et al. Acute febrile neutrophilicdermatosis (Sweet's syndrome) in a child, associated with a rotavirus infection: a case report. J Med Case Rep 2010; 20; 4: 281.

10. Aldemir-Kocabaş B, Karbuz A, Ciftçi E, Demir M, Ince $\mathrm{E}$. An unusual cause of secondary capillary leak syndrome in a child: Rotavirus diarrhea. Turk J Pediatr 2013; 55: 90-93.

11. Johansen K, Hedlund KO, Zweygberg-Wirgart B, Bennet R. Complications attributable to rotavirus-induced diarrhoea in a Swedish paediatric population: Report 
from an 11-year surveillance. Scand J Infect Dis 2008; 40: 958-964.

12. Ciftçi E, Tapisiz A, Ozdemir $\mathrm{H}$, et al. Bacteraemia and candidaemia: a considerable and underestimated complication of severe rotavirus gastroenteritis. Scand J Infect Dis 2009; 41: 857-861.

13. Kaiser P, Borte M, Zimmer KP, Huppertz HI. Complications in hospitalized children with acute gastroenteritis caused by rotavirus: a retrospective analysis. Eur J Pediatr 2012; 171: 337-345.

14. Le Saux N, Bettinger JA, Halperin SA, et al. Substantial morbidity for hospitalized children with communityacquired rotavirus infections: 2005-2007 IMPACT surveillance in Canadian hospitals. Pediatr Infect Dis J 2010; 29: 879-882.

15. Bruijning-Verhagen, Sankatsing V, Kunst A, et al. Rotavirus-related hospitalizations are responsible for high seasonal peaks in all-cause pediatric hospitalizations. Pediatr Infect Dis J 2012; 31: 244-249.

16. Nakatani K, Koizumi H, Nagano R. Septicemia complicating rotavirus gastroenteritis in 5-year-old child. Pediatr Infect Dis J 2009; 28: 555-556.

17. Carneiro NB, Diniz-Santos DR, Amorim C, et al. Septic shock complicating acute rotavirus-associated diarrhea. Pediatr Infect Dis J 2006; 25: 571-572.
18. Scheier E, Aviner S. Septicemia following rotavirus gastroenteritis. Isr Med Assoc J 2013; 15: 166-169.

19. Kashiwagi Y, Sato S, Nakamura M, et al. Klebsiella oxytoca septicemia complicating rotavirus-associated acute diarrhea. Pediatr Infect Dis J 2007; 26: 191-192.

20. González-Carretero P, Noguera A, Fortuny C. Rotavirus gastroenteritis leading to secondary bacteremia in previously healthy infants. Pediatrics 2006; 118: 22552256.

21. Durmaz R, Kalaycioglu AT, Acar S, et al. Prevalence of rotavirus genotypes in children younger than 5 years of age before the introduction of a universal rotavirus vaccination program: report of rotavirus surveillance in Turkey. PLoS One 2014; 9: e113674.

22. Linhares AC, Verstraeten T, Wolleswinkel-van den Bosch J, Clemens R, Breuer T. Rotavirus serotype G9 is associated with more-severe disease in Latin America. Clin Infect Dis 2006: 312-314.

23. Aldemir-Kocabaş B, Karbuz A, Kara TT, et al. Staphylococcus aureus endocarditis and pyomyositis: Rare complications of rotavirus gastroenteritis. Pediatr Int 2015; 57: 780-782.

24. Yang ST, Lin LH, Wu HM. Clinical characteristics of rotavirus gastroenteritis in children in a medical center. Pediatr Neonatol 2010; 51: 112-115. 